\title{
Accident Detection Traffic Light System with Dynamic Fuzzy Logic Control Using FuzzyTech Program and iTraffic Simulation
}

\author{
Faisal AlAwadhi', Mohammed Ali Yousef ${ }^{2}$, Abdulrahman Al-Kandari ${ }^{3}$ \\ ${ }^{1}$ Electrical Power Department, High Institute of Energy, PAAET, Kuwait \\ fa.alawadhi@paaet.edu.kw \\ ${ }^{2}$ Computer Department, Basic Education Collage, PAAET, Kuwait \\ ma. youseflapaet.edu. kw \\ ${ }^{3}$ Computer Department, Basic Education College, PAAET, Kuwait \\ aam.alkandari@paaet.edu.kw
}

\begin{abstract}
The development of traffic signal control systems has become one of the most important topics in this era. Traffic light controllers need to be improved continuously to solve the traffic problems. This paper discussed the proposed hybrid system and demonstrated how the system works from the beginning of the first flag "decrease of cross ratio" until the end of the action system. The proposed system was divided into three main parts: The proposed algorithm (Dynamic Webster with dynamic (ycle Time), Accident Detection System using fuzzy logic theory and Action System depending on Detection System. The focus of this paper is to discuss the accident detection system of the proposed hybrid system, which depended on fuzzy logic and its components. This paper also presented the results of FuzzyTech Software with different scenarios plotting the inputs outputs and the showcases the 3D plot for each one of them for detecting the accident. In addition, it presented results to measure the False Alarm Rate the Accident Detection Rate using FuzzyTech program.
\end{abstract}

Keywords- Intelligent traffic control system, Fuzzy Logic theory, Fuzzy Logic with Accident Detection, Linguistic variables, Membership functions, FuzzyTech Software, False Alarm Rate, Accident Detection Rate.

\section{INTRODUCTION}

Traffic is a major concern for most of the metropolitan cities of the world. Efficient traffic management can have a major impact on the country's economy [1]. The existing methods of controlling traffic are in need of improvement and development [2]. Developing a sophisticated traffic monitoring and control system would result in an effective solution to the traffic congestion problem. Many current traffic light controllers are based on the 'time-of-the-day' scheme. These automated systems do not provide an optimal control for fluctuating traffic volumes [3].

The proposed system gave solution to improve the performance of the traffic system and to minimize delays by using Fuzzy Logic technique. The fuzzy logic control system was modified by Kosonen [4] in which the control agents were given the decision making capability. The Fuzzy Logic system is composed of three main components, the Linguistic variables, the membership functions and the fuzzy rules. The beauty of fuzzy logic is that it allows fuzzy terms and conditions such as "heavy", "less", and "longer" to be quantized and understood by the computer and it allows the implementation of real-life rules similar to the way humans would think [5]. Furthermore, it leads to simplify the rules, make decisions robust and make the right decisions based on observations or common sense even [6].

The main purpose of using fuzzy logic is to avoid faulty inputs and limit the amount of processing required to produce meaningful output in the required time period. Estimation of an optimal queue length has driven lot of efforts in traffic engineering studies. Different techniques exist to determine lengths of the queue in each lane on street width and the number of vehicles that are expected at a given time of day. Fathy el al [7] proposed a queue detection algorithm based on motion detection and vehicle detection algorithm.

The proposed hybrid system has two more components namely the Accident Detection System and Action System. Accident Detection System helps to measure if there is a disturbance in traffic flow by using Fuzzy Logic Theory, to detect and sense the exact location of the incident on the road. It has three phases, each specialize in raising a flag against a road condition. Detection system gathers all required information about the accident and moves this information to the action system where two actions are executed, one against the downstream and the other against the upstream.

The result of this research was presented using FuzzyTech program to simulate the fuzzy system and to prove that the system produced optimal results. 


\section{BACKGROUND}

Arora and Banga [8] have developed an intelligent traffic control system and optimizing traffic control algorithms in their research. They discussed the traditional method for traffic control, which uses a fixed time controller and explained that it does not have the flexibility of modification on real time basis. In their research, they explained different techniques for traffic light control. They also discussed the traffic light control based on image processing and the fuzzy logic controller. Image processing measures the traffic density on the road and according to the traffic density measurements using morphological edge detection method. However, the cost is high due to the use of a high quality camera for intelligent traffic light control. The researchers also highlighted the implementation of fuzzy logic controller and discussed that the fuzzy logic technology has the capability to mimic the human intelligence for controlling the traffic flow.

Antunović and Glavaš [9], on the other hand, discussed the intelligent control in road traffic intersections to be an alternative to the traditional traffic control for more significant improvement in the traffic flow and decreasing the total waiting time of vehicles. They explained that the traditional traffic control is based on preset signal timings, thus non-responsive to real-time fluctuations in traffic demand. They also presented the design and evaluation of a fuzzy logic traffic signal controller for a signalized isolated T-type intersection with proposed improved signal phases. This paper indicated up to 63 percent decrease of total idle time for a typical daily traffic flow.

\section{Accident Detection/Action System MAP}

Fuzzy Logic system is the backbone of the accident detection system. It is responsible of collecting data related to zones, lanes and cross ratio and then acting upon it. What the system requires as an input is the number of cars in each zone and the speed of cars at certain lanes, the rest is concluded from there. The positioning of the sensors is as described in Figure 1. The input of such system can accumulate at certain times and on four ways junction having each controller talk to the other and then to the traffic light to reach a solution can become troublesome. That is one of the reasons why Fuzzy Logic was used. It provides quick decisions to huge amount of uncertain or incomplete data. This ability alone makes fuzzy logic system worthy of adapting and improving on the long run. Fuzzy logic system for accident detection has number of subsystems or processes that interact with each other. Those interrelated processes produces outputs that feed other parts of the system and take actions that affect other processes as well. To say that the system has a central core is imprecise, since if one output fails to be produced the rest of the system will suffer and quite possibly will not function correctly.

This section is a breakdown of the main processes; it provides details about how the system functions. At first, a list of definitions of the terms used in this section is introduced to remove or reduce ambiguity. Then a general walkthrough is given to identify the primary parts of the system. Later, detail description of the process specification and example will be given to link the words to a solid ground that reader can relate to. A flowchart is provided with each section to demonstrate the process in a visual way. Then if needed, a demo of how the subsystem works is provided. The sub processes has been given a different colors as well to make it easier to track dataflow to other subsystems.

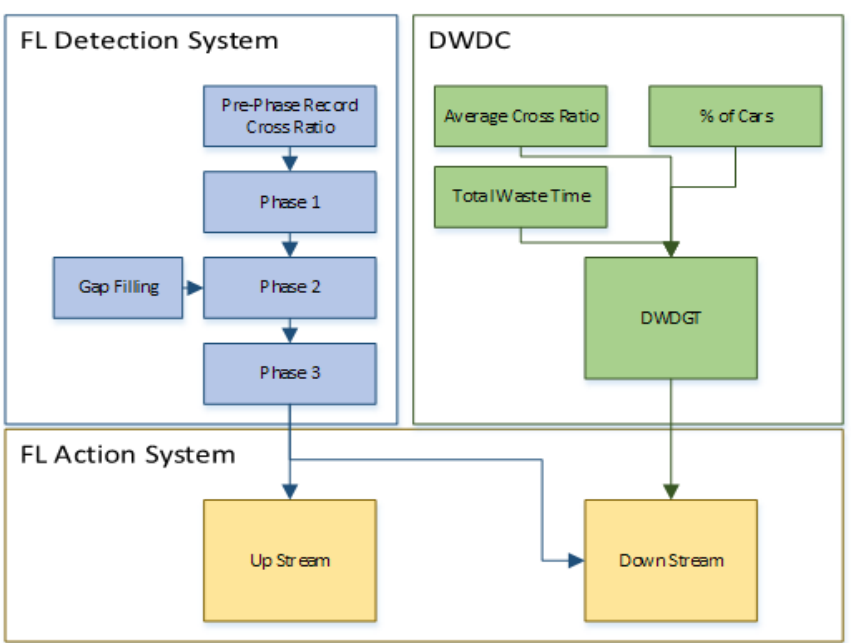

Figure 1 Accident Detection Map (detailed)

\section{EXPERIMENTAL RESULT}

\section{A. Accident Detection and Action Using Fuzzy Tech}

In this section the results of FuzzyTech Software will be discussed. The inputs and outputs will be specified first then the 3D plots will be explained thoroughly.

\section{1) Fuzzy Tech Map}

The following diagram shows how the fuzzy logic system is implemented. The solo output of the system must always be the new effective road, which is understood by the traffic light system afterwards. The map shows different components, each can be related to a specific part of the road. For example, accident zone term relates the zone where the accident happened. The modules used to represent the components are as follows: 
a) Variable groups.

It contains all inputs and outputs of the system. Some of the outputs in the variable group are intermediates. That means, they are inputs into another rule blocks, except the final output which is the new effective road.

b) Rule Blocks

The best way to represent rules related to a certain group of inputs and outputs are rule blocks. Each rule block contains number of rules that connects the different inputs with exactly one output. The rules govern the entire system and can be tweaked to improve the system outputs. Each rule block contains inputs, outputs and the rules spreadsheet. Figure 2 shows the entire Fuzzy Tech map.

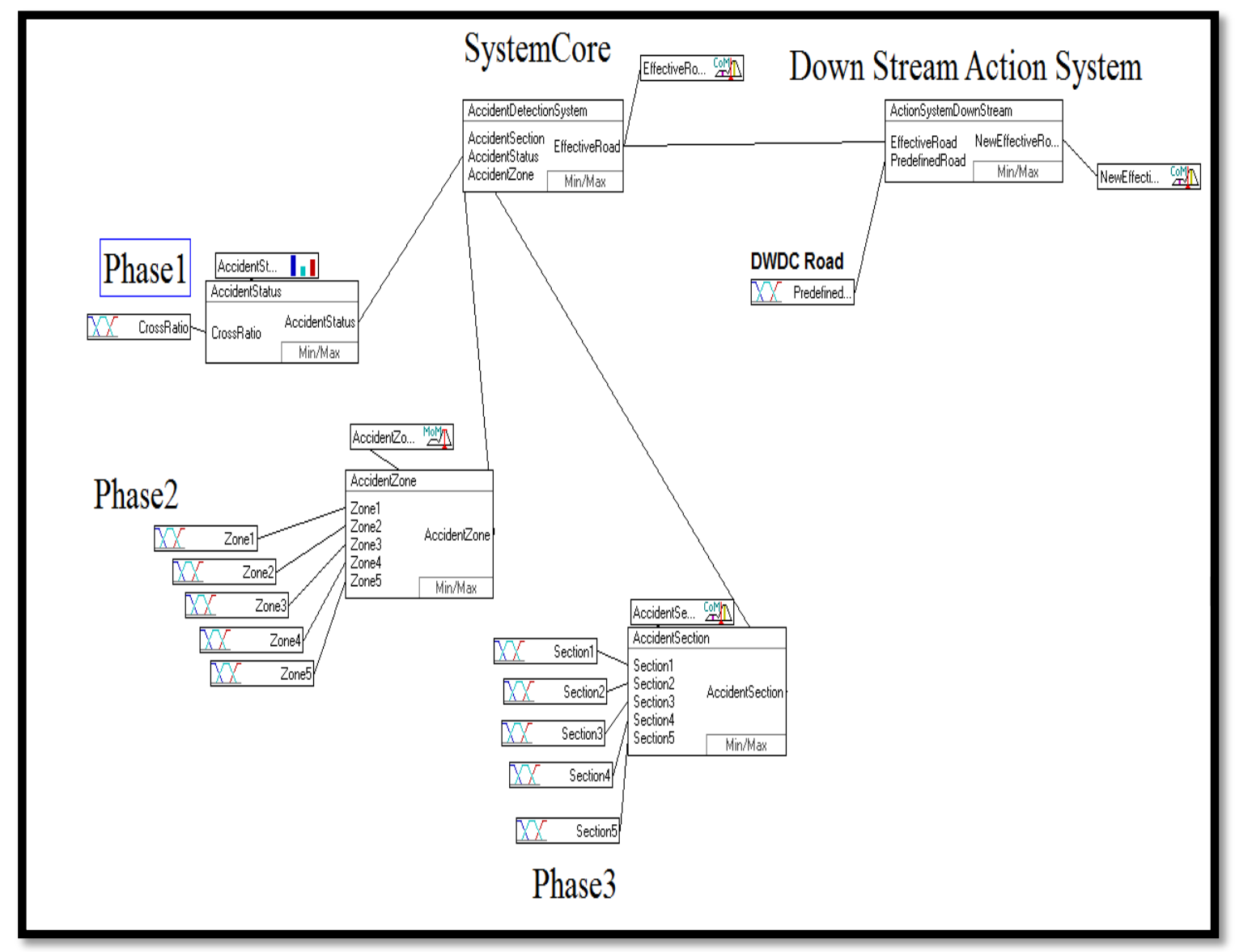

Figure 2 Fuzzy Tech Map

The elements of the Fuzzy Tech map connect in a hierarchical way. The roots are the main phases of the Fuzzy logic system described earlier which are:

c) Phase 1: Accident Status.

d) Phase 2: Accident Zone.

e) Phase 3: Accident Section.

After the roots comes the system core (Accident Detection System) which makes sense of whatever output resulted from each phase. Finally, the Down Stream Action System (Action System Down Stream) which compares the Fuzzy Logic output with the output from DWDC.
Table 1 Inputs of FuzzyTech.

\begin{tabular}{|c|c|}
\hline Name & Description \\
\hline Cross Ratio & The number of cars crossing each second. \\
\hline Section 1 & \multirow{5}{*}{$\begin{array}{l}\text { The speed of cars in the corresponding } \\
\text { section. }\end{array}$} \\
\hline Section 2 & \\
\hline Section 3 & \\
\hline Section 4 & \\
\hline Section 5 & \\
\hline Zone 1 & \multirow{5}{*}{$\begin{array}{l}\text { The number of cars in the corresponding } \\
\text { zone. }\end{array}$} \\
\hline Zone 2 & \\
\hline Zone 3 & \\
\hline Zone 4 & \\
\hline Zone 5 & \\
\hline Predefined Road & The road captured from DWDC system. \\
\hline
\end{tabular}


2) Complete List of Inputs, Outputs and Intermediates in Fuzzy Tech

Before discussing the different scenarios, Table 1 above shows a complete list of the inputs and outputs in the system alongside a brief description of each.

TABLE 2

INTERMEDIATES OF FUZZYTECH.

\begin{tabular}{|l|l|}
\hline Name & Description \\
\hline Accident Status & $\begin{array}{l}\text { The probability of the accident } \\
\text { in the corresponding road. }\end{array}$ \\
\hline Accident Section & $\begin{array}{l}\text { The location of the accident on } \\
\text { a section scale. }\end{array}$ \\
\hline Accident Zone & $\begin{array}{l}\text { The location of the accident on } \\
\text { a zone scale. }\end{array}$ \\
\hline Effective Road & $\begin{array}{l}\text { The road determined from the } \\
\text { Current system, the Fuzzy Logic } \\
\text { system. }\end{array}$ \\
\hline
\end{tabular}

TABLE 3

OUTPUTS OF FUZZYTECH.

\begin{tabular}{|l|l|}
\hline Name & Description \\
\hline New Effective Road & $\begin{array}{l}\text { The final road determined by } \\
\text { the Fuzzy Logic System. }\end{array}$ \\
\hline
\end{tabular}

3) Quick Review of Zone Status and Section Status Spreadsheet Rules

The rules spreadsheet is what makes the FuzzyTech system refer to. Thus, in order to get a brief understanding on how the rules work a table and figures are displayed to explain the potential scenarios that are actually covered by the system. The researcher aware that there are a lot of scenarios available, however this paper will focus on presenting the most common ones.

a) The Default Road Rules:

The default road is basically one with a normal distribution of cars. This road in case of an accident or not have the following characteristics:

i. The zones have a gradual distribution of cars.

ii. The accident zone is normally between two high density, congested zones.

iii. The difference between the zones is of 1 fuzzy term not more.

Examples of such a road are demonstrated in the tables below (The red line represent the traffic light side):

TABLE 4

No accident Default Road.

\begin{tabular}{|l|l|l|l|l|}
\hline & & & & \\
\hline & & & & \\
\hline & & & & \\
\hline & & & & \\
\hline & & & & \\
\hline 50 cars 50 cars 40 cars Empty Empty
\end{tabular}

TABLE 5

No aCCIDENT DEFAULT ROAD 2.

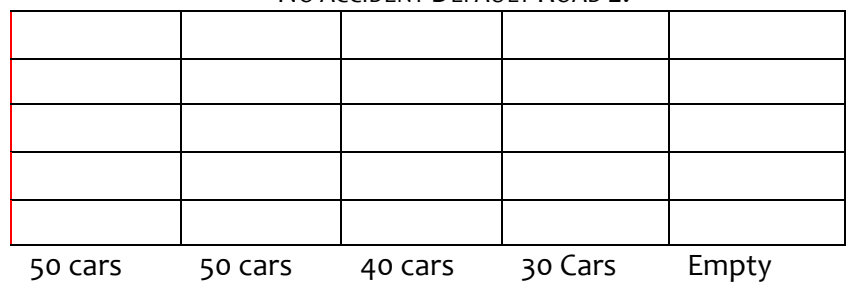

TABLE 6

ACCident ENABLEd Default Road.

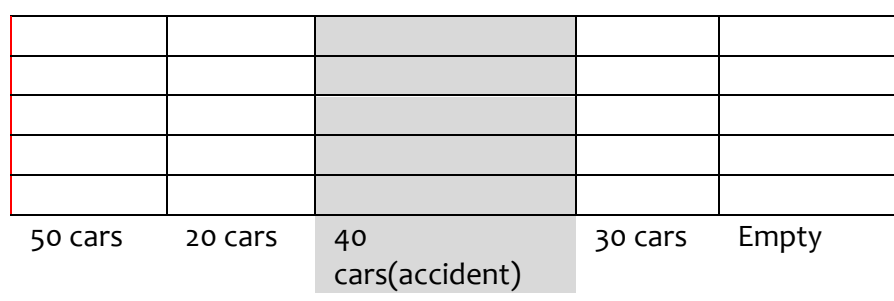

TABLE 7

Accident Enabled Default Road 2.

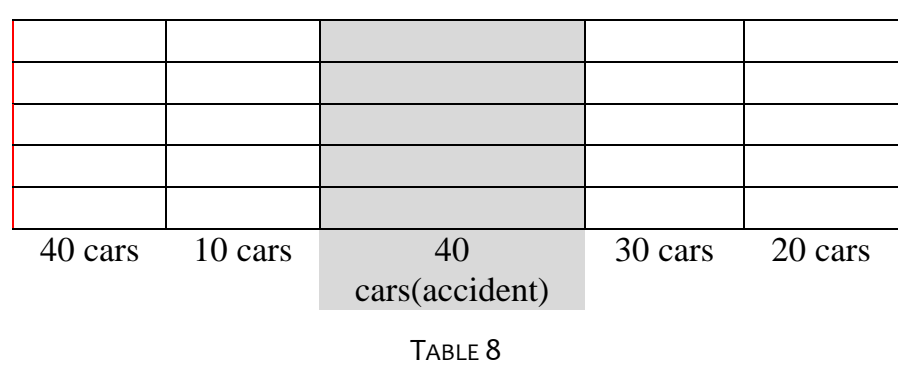

Accident Enabled Default Road 3.

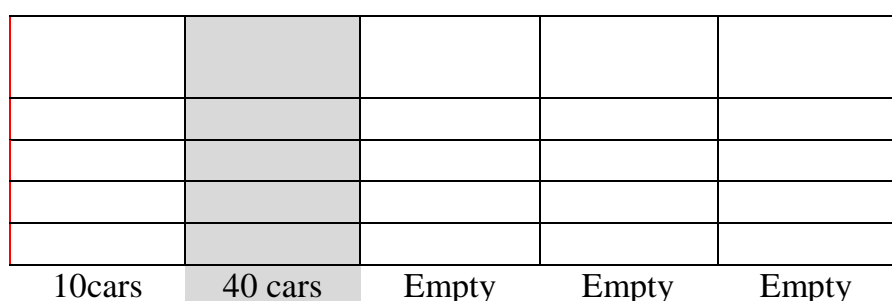

4) Scenario 1:

In this scenario the main focus will be Zone 5 which is the accident zone and section 3 which is the accident section.

a) Inputs/Outputs

The cross ratio has been set to 1 car per second, which is very slow. The predefined road is 2 and half zones long. As for the road conditions, Section 3 is the slowest one among other sections. Unlike scenario 1, where sections had somewhat close values, in the scenario section 3 is very slow and therefore the output of Section Status does not show any fractions like half or quarter. Zones however are much closer to each other in term of cars. The difference between Zone 1 and Zone 2 is smaller than the difference between Zone 4 and Zone 5 making Zone 5 
the better candidate for accident zone as seen in the output.

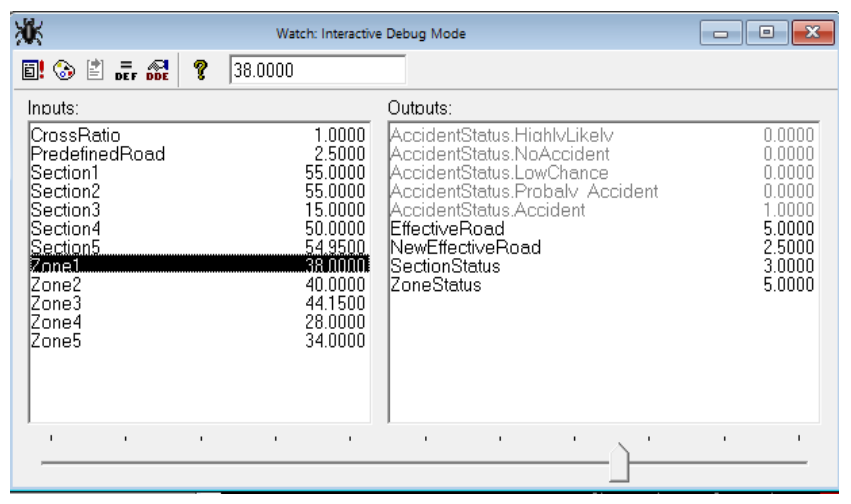

Figure 3 Inputs and outputs of Scenario 1.

There are other different scenarios as well to deal with patterns similar to this one and all of them can be implemented in the Rules spreadsheet.

b) 3 D Plot

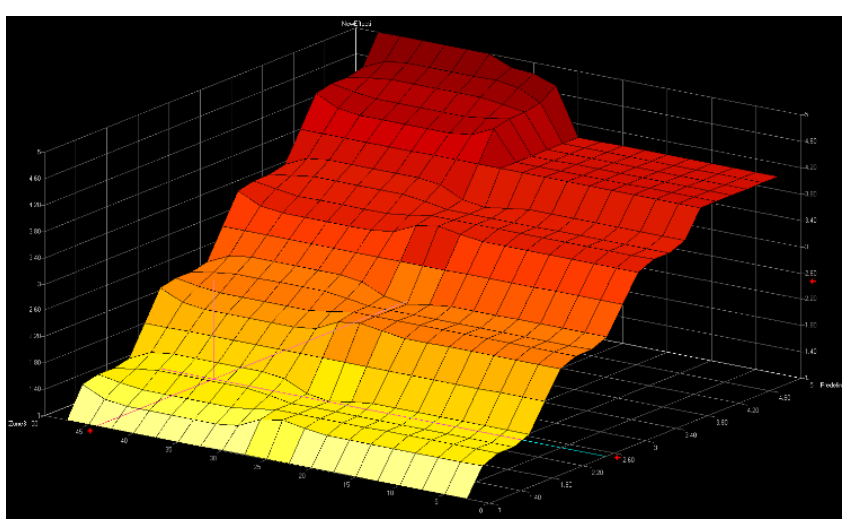

Figure 4 3D Plot of scenario 1.

In the $3 \mathrm{D}$ plot Figure $4, \mathrm{X}$-axis is Zone $3, \mathrm{Y}$-axis is Predefined Road and Z-axis is New Effective Road. Like in the previous scenario, the zone is not affected by the predefined road. A major difference though is the number of cars relationship with the new effective road. There are two types of increase happening on the graph, the first being crisp and the second being continues and smooth. The first type can be noticed from 25 cars and less then it is similar to that exist in scenario 1. This type of increase is normal and as the previous example it stops after the predefined road becomes longer the effective road. The other type of increase in the graph begins after the mark of 25 cars. This increase indicates a stronger relationship between the predefined road and the new effective road (almost linear). It also scales to the point of more than just 3 zones to 5 zones; this means that if the predefined road increases then the new effective road will increase indicating that the effective road is actually 5 zones which is indeed the case. The reason why the new effective road does not scale to 5 zones if the number of cars is less than 25 is that the accident zone will be shifted from Zone 5 (where the gap is Zone 4) to Zone 4 (where the gap is Zone 3) because the number of cars dropped to 25 which is the threshold of the accident zone in this scenario.

\section{5) Scenario 2:}

In this scenario Zone 3 and 5 are the main suspects of accident zones. Section 3 is the slowest section and therefore the one holding the accident.
a) Inputs/outputs

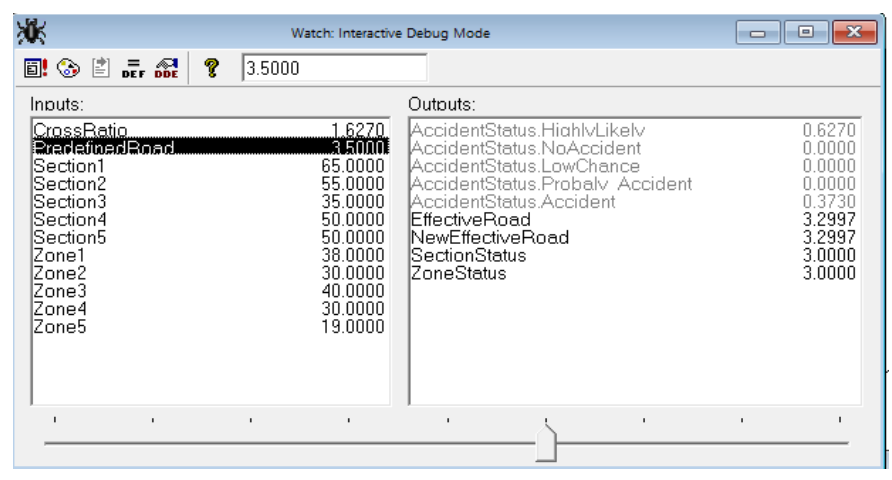

Figure 5 Inputs and Outputs of scenario 2.

In this scenario the Zones have a tricky setup. There are two zones with low number of cars and similar pattern to accident zones, namely Zone 2 and 4. The gaps are indicators of accidents in the previous zones but the replication of the pattern can confuse the FuzzyTech system. As a solution the FuzzyTech system picks and effective road that is in-between Zone 3 and 5. In other cases the effective road can differ or swing to the zone that is most likely to be the one holding the accident. The system decides depending on the Degree of Membership between the different zones. Zones with higher degrees are more likely to be picked or preferred to the other zones.

$$
\text { b) } 3 \text { D Plot }
$$

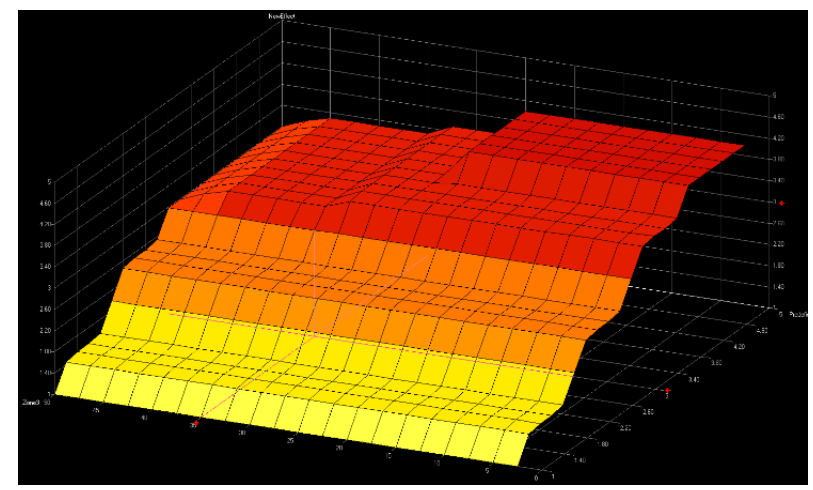

Figure 6 3D Plot of scenario 2 
In the $3 \mathrm{D}$ plot Figure 6, $\mathrm{X}$-axis is Zone $3, \mathrm{Y}$-axis is Predefined Road and Z-axis is New Effective Road. In this plot the number of cars in Zone 3 is irrelevant to the length of the predefined road. The number of cars in Zone 3 however affects greatly the new effective road length. The more cars in Zone 3 the shorter the new effective road gets. The fewer cars in Zone 3 the longer the new effective road gets. This is due to the swing discussed earlier on in the inputs and outputs as there are two zones with similar accident status chance. Once Zone 3 has less cars then it is more likely not having an accident (because it turns into a gap) which means that the other zone (zone 5) will be the one holding the accident. The increase of the predefined road is coupled with an increase of the new effective road on some parts of the graph. Those parts are when the number of cars in Zone 3 is less than 25 cars. The new effective road will increase as well as the predefined road will be exactly identical to the effective road (Zone 5). Otherwise, when the number of cars is more than 25 cars, the increase stops at Zone 3 which is the maximum new effective road in this case (as the system will pick the shorter road always hence the zone 3 instead of zone 5).

\section{B. Using Itraffic to Measure False Alarm Rate}

One of the attributes that define a good algorithm from a bad algorithm is how often does it produces faulty results. In iTraffic system simulation, the software provides the researcher an easy way to conduct experiments on a very large scale of scenarios with different variables and without the need to actually implement the system, monitor it and test it on real roads, as it will be both costly and risky or unsafe. The software provides the ability to generate random accidents on different roads. These accidents will eventually cause some sort of disturbance from which the software will conclude using the detection algorithm that something went wrong and on those basis, starts checking for accidents on the road. The options available to control the accidents' generator as can be seen in Figure 7 are:

a) Accident Interval: The time gap between each accident and the other.

b) Accident count: Total number of accidents to be generated in this session.

c) Type of accidents distribution:

d) Random: Randomly pick roads, sections and zones for each accident.

e) Specific: Define exactly where to place the accident according to the road, track "zone" and sensor "section".

f) Action system: This option indicates whether the action system should react to the accident or not.

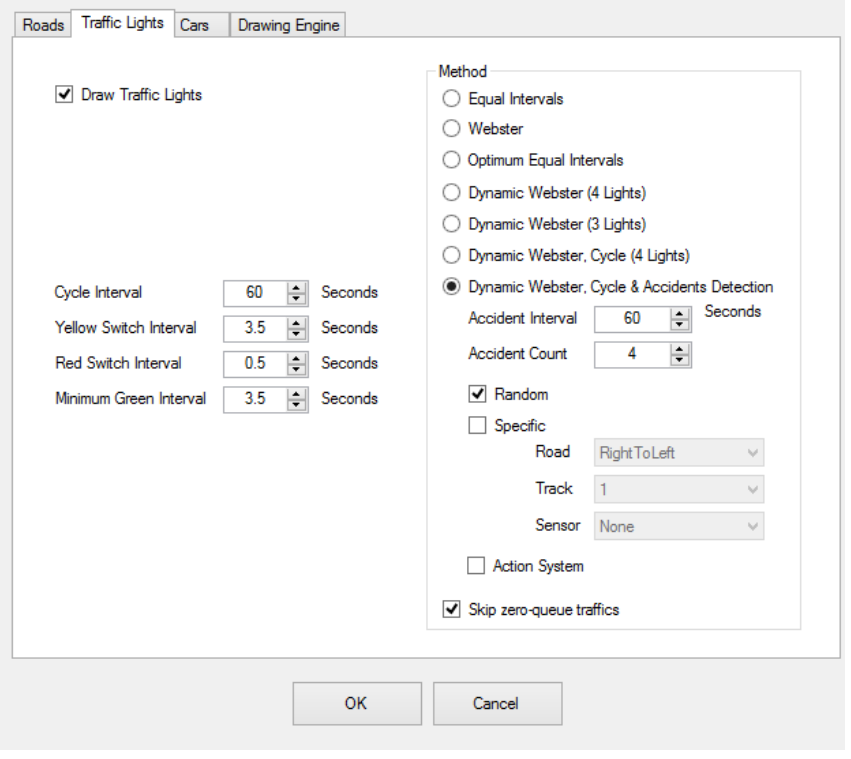

Figure 7 Accident generator options in iTraffic

Once the options are set, the software can start detecting accident alongside executing the selected algorithm, which is for now DWDC. It is important to notice that the flow rate of cars with or without the accident generator is steady.

In the Figure 8 , an accident is generated in the RTL road. The car responsible for the congestion is the red car marked with the circle. Notice that cars behind the accident car are yet to move or respond to the accident. This can take at more than one cycle as new cars tend to line up and get delayed as well only to realize late that there is indeed an accident.

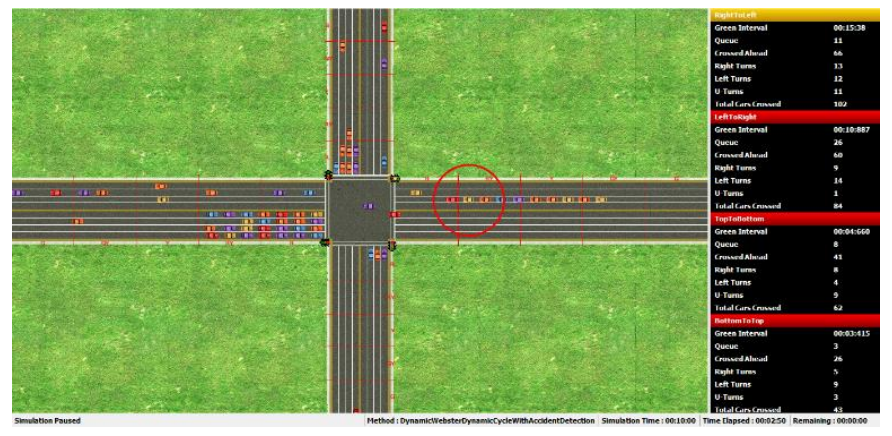

Figure 8 Car Accident in iTraffic simulation

Figure 9 shows another scenario of an accident where the orange car malfunctioned and stopped moving in the LTR road. The ability to control when and where the accident happen provides more flexibility which result in better control of the rules that decide if an accident had happened or not. 


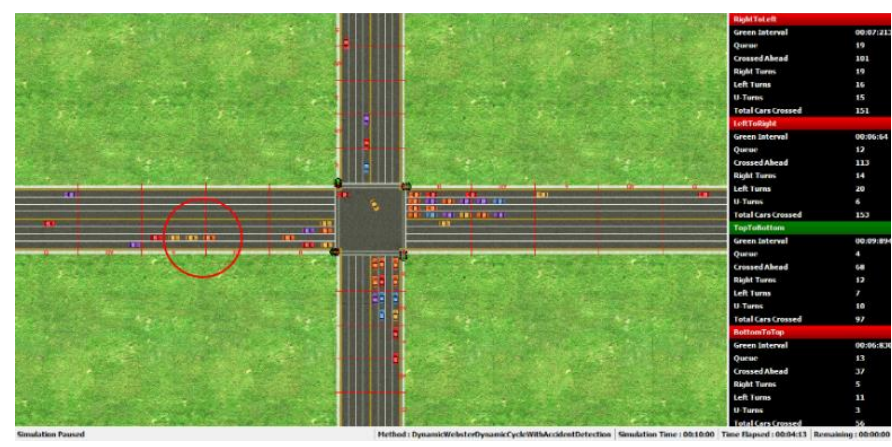

Figure 9 Car Accident 2 in iTraffic simulation

High false alarm rates are attributed to the fact that the detectors are the only source of input with no way of validation (visual or human). Therefore if any of detectors were to malfunction in real life for example, it would cause a high false-alarm rate. In addition, false alarm rates depends on the threshold alone tend to fail occasionally, as the road is a richer environment where other factors can affect the reading of the sensors. This leads to more experiments being conducted with different cycle intervals "as before with methods comparison". Each time, the cycle interval is changed while other factors remain the same. The decisive factor is the cycle interval time, which is an increment of 30 seconds from 60 to 330 seconds: $60,90,120,150,180,210,240,270,300$ and 330 . All of this leaves us with a total of 100 accidents in the entire experiment and random accident location.

The following table demonstrates the new constants:

TABLE 9

NEW CONSTANTS.

\begin{tabular}{|l|l|}
\hline Option & Value \\
\hline Duration of the Accident & 60 Seconds \\
\hline Number of Accidents & 10 \\
\hline Accident location & Random \\
\hline
\end{tabular}

There are only two new definitions for this simulation test:

- $\quad$ FAR: False Alarm Rate

- $\quad$ ADR: Accident Detection Rate

- $\quad F A R+A D R=1$

System Outputs: The new outputs to be measured are:

- The number of false alarms $=4$ times out of 100

- $\quad$ The number of successful detections $=96$ times out of 100

- $\quad \operatorname{FAR}(0.04)+\operatorname{ADR}(0.96)=1$

\section{CONCLUSION}

This paper discussed two separate systems that construct the main proposed hybrid system, that is the Detection system and the Action system. This paper focused on Detection system and demonstrated how the system works from the beginning of the first flag "decrease of cross ratio" until the end of the action system. Fuzzy Logic system is part of the detection system and contributes in the action taken. Detection system helps to measure if there is a disturbance in traffic flow of real time detection, with exact location of cross ratio must be monitored. There are three phases of the system which were discussed in this paper. The first phase is concerned with the cross ratio, the second phase is concerned with the zones of the road and the third phase is concerned with the sections. The results of FuzzyTech Software with different scenarios plotting the inputs and outputs were also presented in this paper.

\section{REFERENCES}

[1] Bilal, Jubair Mohammed, and Don Jacob. "Intelligent traffic control system." Signal Processing and Communications, 2007. ICSPC 2007. IEEE International Conference on. IEEE, 2007.

[2] Lemelson, Jerome $H$., et al. "Intelligent traffic control and warning system and method." U.S. Patent No. 6,633,238. 14 Oct. 2003.

[3] Kulkarni, G. H., and P. G. Waingankar. "Fuzzy logic based traffic light controller." Industrial and Information Systems, 2007. ICIIS 2007. International Conference on. IEEE, 2007.

[4] Kosonen, I. (2003). Multi-agent fuzzy signal control based on realtime simulation. Transportation Research Part C: Emerging Technologies, 11(5), 389-403.

[5] Artificial Intelligence Center and Jalan Semarak. "Intelligent traffic lights control by fuzzy logic." Malaysian Journal of Computer Science 9.2 (1996): 29-35.

[6] Wiering, M. A., Veenen, J. V., Vreeken, J., \& Koopman, A. C. M. (2004). Intelligent traffic light control. UU-CS, (2004-029).

[7] Fathy, M., \& Siyal, M. (1995). Real-time image processing approach to measure traffic queue parameters. Paper presented at the Vision, Image and Signal Processing, IEE Proceedings-.

[8] Arora, Madhavi, and V. K. Banga. "Intelligent Traffic Light Control System using Morphological Edge Detection and Fuzzy Logic." International Conference on Intelligent Computational Systems (ICICS'2012) Jan. 7-8, 2012 Dubai

[9] Mladen Antunović, and Hrvoje Glavaš. "Fuzzy Logic Approach for Traffic Signals Control of an Isolated Intersection." 25. Skup o prometnim sustavima $s$ međunarodnim sudjelovanjem AUTOMATIZACIJA U PROMETU 2005 (2005). 